\title{
Reconstruyendo el camino del proceso de enseñanza-aprendizaje de la Solución de los sistemas de ecuaciones lineales
}

\author{
Reconstructing the path of the teaching-learning process of the Solution \\ of the systems of linear equations
}

\author{
Elisain Medina-Sandoval ${ }^{\mathrm{a}}$ \\ ${ }^{a *}$ Estudiante de licenciatura en matemáticas, Universidad Francisco De Paula Santander, orcid 0000-0003-4706-4274, elisainms@ufps. \\ edu.co, Cúcuta, Colombia
}

Forma de citar: Medina-andoval, E. Reconstruyendo el camino del proceso de enseñanza-aprendizaje de la Solución de los sistemas de ecuaciones lineales. Eco Matemático, 10 (2), 79-88

Recibido: 12 de marzo de 2019

Aceptado: 16 de mayo de 2019

\section{Palabras clave}

Algebra, didáctica de las matemáticas Ecuaciones lineales, educación matemática.

*Autor para correspondencia: elisainms@ufps.edu.co

Resumen:La solución de los sistemas de ecuaciones lineales es una temática fundamental, no solo en el área de las matemáticas, sino también de otros campos del conocimiento, por lo cual este estudio se centró en describir el proceso de enseñanza-aprendizaje de esta temática, para esto se realizó una revisión documental, donde los artículos encontrados se clasificaron en tres grupos: caracterización de la temática, errores y dificultades en el proceso de enseñanza-aprendizaje del tema y también la educación matemática tanto a nivel general, como en el área del algebra, la cual se abordó con el fin de tener un marco mayor en cuanto al proceso educativo de los estudiantes. De esta forma se determinó que algunas de las falencias se deben a la falta de conceptualización en la parte algebraica, por lo cual se propone formación en esta área, desde la educación primaria, al mismo tiempo se promueve la implementación de nuevas estrategias y herramientas didácticas en el desarrollo del tema. 


\section{Keywords}

Algebra, didactics of mathematics, linear equations, mathematical education.

\begin{abstract}
The solution of linear equation systems is a fundamental issue, not only in the area of mathematics, but also in other fields of knowledge, so this study focused on describing the teaching-learning process of this subject, for this a documentary review was conducted, where the articles found were classified into three groups: characterization of the subject, errors and difficulties in the teaching-learning process of the subject and also mathematical education both at the general level and in the area of algebra, which was addressed in order to have a greater framework in terms of the educational process of the students. In this way it was determined that some of the deficiencies are due to the lack of conceptualization in the algebraic part, for which training in this area is proposed, from primary education, at the same time it promotes the implementation of new strategies and didactic tools in the development of the subject.
\end{abstract}

\section{Introducción}

Durante el desarrollo de la humanidad las ecuaciones lineales han sido de gran importancia para los seres humanos debido a que su solución ha permitido dar respuesta a diferentes problemáticas que ha padecido el hombre. Tal es el caso de las ecuaciones de primer grado que fueron empleadas para resolver dificultades por culturas como la babilónica, la egipcia y la griega y que cuyos métodos y resultados han sido útiles a lo largo de la historia e incluso en la actualidad (Dalcin y Olave, 2007).

Ahora bien, actualmente los sistemas de ecuaciones lineales es una temática que es abordada durante la educación Básica Secundaria, el cual en la mayoría de los casos se desarrolla de la forma en que la plantea el siguiente autor, donde:

La atención se sitúa principalmente en el aprendizaje de los métodos de resolución, en la ejercitación de los mismos y para finalizar la enseñanza del tema, se proponen problemas en lenguaje verbal que pueden resolverse traduciendo la información que da el enunciado de dichos problemas a través de un sistema de ecuaciones. (Ochoviet, 2009, pg 11).
Así pues, como lo especifico dicho autor, se ha establecido un proceso de enseñanza el cual es orientado por el docente, pero, aunque tenga un esquema casi que preestablecido, se deja al profesor la labor de realizar la planeación y ejecución de sus clases, en las cuales ha de desarrollar diferentes estrategias de enseñanza, para lograr que la temática quede lo más clara posible (Abrate, Font y Pochulu, 2008).

Sin embargo, esta no es la única cara de la moneda, ya que desde el lugar del estudiante existen otros factores que entran a jugar a la hora del proceso de aprendizaje, entre estos se destacan, las dificultades matemáticas en cuanto a conceptos y comprensión de las mismas, así mismo también es relevante los errores que los aprendices tienen en el desarrollo de la temática (Benítez y Drouhard, 2015). Así pues, es labor del docente conocer y cooperar en la mejora de estos factores para lograr la claridad de la temática.

De esta forma hay un amplio panorama que conocer desde la perspectiva del proceso de enseñanza-aprendizaje de la solución de los sistemas de ecuaciones lineales. 


\section{Materiales y métodos}

El presente estudio se consolida como una revisión documental, donde se identificaron características entorno a una temática en particular, con el fin de demarcar lo relevante de tal tema (Valencia, 2012).

Como ya se mencionó anteriormente el centro del estudio es la solución de los sistemas ecuaciones lineales, de esta forma como criterio de búsqueda se tuvo en cuenta que los documentos encontrados abordaran cualquiera de las siguientes dimensiones: caracterización de la temática, errores y dificultades en el proceso de enseñanza-aprendizaje de la temática y también la educación matemática tanto a nivel general, como en el área del algebra, la cual se abordó con el fin de tener un marco mayor en cuanto al proceso educativo de los estudiantes.

De esta forma se realizó un rastreo en varias bases de datos, entre las cuales se encuentran scopus, gale, google scholar, scielo, science direct. Allí se consultaron un total de 55 artículos, de los cuales se descartaron 5, ya que no contribuían a las dimensiones de análisis. Por otro lado, la búsqueda se realizó entre el 26 de febrero al 3 de abril del 2019, y se encontraron artículos publicados a partir del año 2001 hasta el 2019.

Por otro lado, se puede decir que la gran mayoría de los textos consultados son en idioma español, sin embargo, se incluyó una minoría en idioma portugués y otros en inglés.

\section{Resultados y análisis}

Como se mencionó anteriormente, los artículos se clasificaron en tres dimensiones, así pues, para una mejor especialización en el abordaje de la temática, el análisis de los resultados se agrupo de la misma forma.

\section{Contextualización de la temática}

La ecuación se obtiene al realizar la igualación de dos expresiones que representan una situación problema y sus elementos se componen de variables cuyos valores se pueden determinar mediante ciertos métodos (Alfonso, García, y Gabarda, 2016).

La conceptualización de los sistemas de ecuaciones lineales, se logra por medio de diferentes tipos de representaciones, que se complementan entre sí (De Herrero, 2004). Al igual que otras temáticas particulares de las matemáticas, los sistemas de ecuaciones lineales, presentan formas de simbolización, y estas son las que facilitan la resolución de los mismos debido a que reducen la complejidad de dichos elementos (Costa, 2010). Los sistemas de ecuaciones lineales se pueden simbolizar como elementos geométricos, por medio de los cuales analíticamente es posible determinar una solución, aun así, para llegar a esta existen más métodos, pero es más pertinente resolverlo de acuerdo a la forma que más se facilite (González y Roa, 2015).

De esta forma es evidente que se llega a la solución de una forma más sencilla, cuando se realizan procesos mentales de nivel superior. En este orden de ideas, un caso particular se evidencia al plasmar las ecuaciones lineales en un plano cartesiano, desde una perspectiva espacial, se facilita la comprensión del lenguaje matemático que estaba inicialmente representado en una simbología abstracta para el estudiante (Jara, Lorca, Lorca, Saldias y Leo, 2019) por otro lado, es necesario tener presente que los sistemas de ecuaciones lineales tienen aplicabilidad en la realidad, en diferentes campos por donde se desenvuelve el hombre, entre ellos el de la economía, donde la ecuación lineal permite la determinación del precio de acuerdo a la variación de la demanda y la oferta (Rodríguez y Fernández, 2010). 


\section{Errores y dificultades en el proceso de enseñanza-aprendizaje de la temática}

\section{Errores}

Se puede decir de forma general que un error es una prueba de que existe alguna dificultad en el dominio de un determinado tema (Pérez, Mantecón, Polo y González, 2019). Así pues, hacen referencia a la llegada del estudiante a un puerto cognitivo equivocado, sin ser necesariamente una carencia o ignorancia del conocimiento (Del Puerto, Minnaard, y Seminara, 2006).

Los errores han de ser utilizados como punto de partida para analizar, y fortalecer la falencia que ha dado pie al mismo, de tal forma no será una razón para descalificar, sino por el contrario para mejorar, al mismo tiempo que se contribuye en la formación de personas integras en cuanto a lo académico, como a lo social, debido a que desarrolla el pensamiento crítico (Sánchez, 2014).

Dentro del rastreo realizados, se encontró que un error frecuente es la utilización incorrecta de los datos, lo cual se debe a una interpretación errónea de la lectura del enunciado del problema o ejercicio (Díaz, 2016). Algunas de estas fallas se evidencian en la falencia que hay en traducir del lenguaje natural al algebraico el enunciado lo cual sucede con indicaciones sencillas, tales como: "el cuádruple de una variable", o "el numero aumentado en cinco unidades" (Ramírez, Chavarría, Barahona, y Mora, 2009).

Por otro lado, se sabe que las representaciones graficas permiten una mejor comprensión de las ecuaciones involucradas en el sistema, sin embargo, es difícil en algunos casos que los estudiantes logren realizar un gráfico adecuado (Córdoba, Díaz, Haye y Montenegro, 2013).

De forma general Es importante realizar un seguimiento, en el cual se registren los errores cometidos por los estudiantes y la cantidad de veces que estos los comenten, para que le docente reflexivamente pueda realizar acciones de mejora al igual que realice modificaciones en sus clases con el fin de evitar posteriores equivocaciones (Iribar y Rouseau, 2009).

\section{Dificultades}

Las dificultades se presentan como obstáculos, que evitan que el estudiante desarrolle su aprendizaje, así pues, estas pueden ser de diferente índole, tal es el caso de que estudiantes manifiestan que se sienten aburridos y desmotivados con las clases, así mismo existen temores en cuanto a la falta de comprensión de las temáticas abordadas (Arroyo, 2014).

Por otro lado, en algunos estudiantes resulta sencillo resolver ecuaciones por medio de despejes u otros procesos, en lugar de realizar planteamientos desde un enunciado dado (Esquinas, 2009). De esta forma se afirma que los problemas presentan mayor dificultad en comparación con los ejercicios procedimentales de solución de sistemas de ecuaciones lineales debido a que en muchas ocasiones se realizar por medio de la comparación con otros similares realizados por el docente, de esta forma se evidencian fallas en la didáctica, lo cual impide una mejor interpretación de los enunciados (Sanjosé, Valenzuela, Fortes, y Solaz-Portolés, 2007).

En este orden de ideas, cuando un estudiante no logra cambiar sus registros de una representación a otra, se evidencia que aún no ha logrado una comprensión de la temática (Model, 2005). Así mismo, es relevante que se comprenda totalmente lo que un símbolo representa de tal forma que el estudiante quede sin lagunas mentales, ya que cuando no se tiene claridad, algunos terminan sumando variables diferentes y llegando a resultados incoherentes (Cambriglia, 2008). 
Ahora bien, en nivel universitario se evidencia que existe falta de comprensión del significado de las letras dentro de las operaciones algebraicas, por lo cual hay deficiencia en el desarrollo pensamiento algebraico de los mismos. (Suárez, Alex y Gómez, 2014).

Así mismo, Se evidencio en estudiantes de primer semestre dificultades por falta de conceptualizaciones algebraicas como lo es el despeje, la simplificación, que a la hora de resolver ecuaciones son altamente necesarias (Castellón, Sánchez, Moreno y Páez, 2003). También existen carencias en traducción del lenguaje, comprensión de conceptos matemáticos, e interpretación de símbolos (Rey y Sastre, 2013). Así como dificultades en la interpretación del valor de las letras como variables o como objetos funcionales. (Alurralde e Ibarra, 2008).

\section{Educación matemática}

Después de haber caracterizado a grandes rasgos lo concerniente a los sistemas de ecuaciones lineales, y posteriormente haber descrito algunos de los errores y dificultades frecuentes en el abordaje del tema, se hace necesario explorar entre las alternativas que ofrece la educación matemática para la orientación de esta temática.

La educación es un proceso donde el hombre además de escolarizarse se prepara para la vida en sus diferentes dimensiones, de esta forma es importante pensar en una formación integral, ya que el hombre se realiza en diferentes roles (Cedro y Moura, 2010). Así pues, se espera que los estudiantes lleguen a ser ciudadanos matemáticamente ilustrados, haciendo referencia a que los seres humanos diariamente nos enfrentamos a situaciones que ponen en juego nuestras capacidades matemáticas, como lo es pensar, contar, calcular, medir, analizar, y demás. De tal forma la educación debe formar a los estudiantes para que puedan optimizar dichas habilidades (Romero, 2004).
De esta forma es relevante que la formación algebraica inicie desde los primeros años de educación primaria (Castro, Godino y Rivas, 2011). El iniciar a estudiar el algebra desde temprana edad permite al estudiante desarrollar sus procesos mentales, así pues, lograra analizar, establecer conjeturas, organizar sus ideas, consolidar argumentos (Kiener, 2015).

Por otro lado, en cuanto a los errores y dificultades abordados en el apartado anterior, se evidencio que la traducción de los enunciados del lenguaje natural al lenguaje matemático es uno de los procesos de mayor dificultad para los estudiantes, por lo cual se allá pertinente el uso de los modelos matemáticos, debido a que son simbolizaciones matemáticas de una situación compleja, por lo cual establecen relación entre los elementos que allí se involucran (Linares, Ramos, Sánchez, Sarabia y Vitoriano, 2001). Asi pues, un modelo mental representa un esquema de conocimientos, de ahí que quien tenga bien establecido un modelo mental de los sistemas de ecuaciones lineales, podrá resolver estos, mediante la adaptación de las variables del problema a dicho modelo (Otero, Papini y Elichiribehety, 2016).

Sin embargo, se puede afirmar que, aunque es importante el uso de la modelación matemática en el aula, se debe buscar un equilibrio entre las temáticas en acción y este proceso mental de tal forma que se logre complementar y así allá una adecuada comprensión de las temáticas (Gaisman, 2009).

Ahora bien, en cuanto al proceso didáctico, se propone que en la escuela se recree el contexto del estudiante de tal forma que este pueda conocer el mundo exterior por medio de esto y dar respuestas por medio de la educación a sus problemas reales, de tal forma que cuando el aprendiz salga de la escuela, sea capaz de aplicar lo anteriormente aprendido (Medrano y Flores, 2018). Así mismo el docente ha de asumir el rol orientador de la clase promoviendo a el estudiante para que investigue, 
pregunte, trabaje, se inquiete, de tal forma que él tome las riendas de su aprendizaje (Duarte, 2013).

En este orden de ideas, se proponen alternativas didácticas para lograr estos propósitos, una de ellas es el trabajo cooperativo, por medio del cual los estudiantes construyen su conocimiento, debido a que allí se plantean actividades que les permite interactuar entre compañeros, en el caso específico de las ecuaciones lineales, se pueden proponer actividades, que permitan dar solución a problemas, así que entre compañeros contribuyan aclarándose dudas y proponiendo opciones, dando espacio a la creatividad y al trabajo en equipo (Martínez, Rincón y Domínguez, 2011). Así mismos, Los aprendices pueden compartir con sus semejantes las estrategias que utilizan para la resolución de problemas, para que así pueden complementar sus aprendizajes y discutir sobre cuál ha de ser la más optima (García 2019).

Por otro lado, en las instituciones educativas se propone ejecutar transversalidad con problemas del entorno, se logra una mejor comprensión de los sistemas de ecuaciones lineales, ya que al presentar situaciones que involucren el tiempo, el dinero $u$ otros elementos que le son familiares a los alumnos, se lograra asociar el nuevo conocimiento con los conocimientos previos, desarrollando así un nuevo aprendizaje (Oaxaca, De la Cruz, Sánchez, 2009).

Un ejemplo de ello es el relacionar el balance de la materia con los sistemas de ecuaciones lineales, donde se pudo evidenciar que se facilitó la comprensión de las dos temáticas, ya que ambas parten del concepto de equilibrio (Trejo y Camarena, 2011).de esta forma es apreciable que, al realizar la transversalización de las matemáticas con otras áreas del conocimiento, se logra el desarrollo del conocimiento más significativo.

Así pues, desde el campo de la tecnología, se evidencia que existen múltiples alternativas que permiten el fortalecimiento de los conocimientos en los estudiantes, como por ejemplo al implementar un programa tecnológico, se evidencio que los estudiantes comenten menos errores en comparación con el papel, así mismo las equivocaciones que tuvieron las corregían por sí mismos, ya que eran bastantes cuidadosos y reflexivos alrededor de estas (Issakova, 2006).

También en otro estudio, se evidencio que al emplear el software GeoGebra, los estudiantes realizaron más rápido y fácilmente sus tareas, así mismo generaron discusión entre las diferencias de sus resultados, en la solución de un sistema (Figueroa Vera, 2013).

Así mismo al aplicar un software especializado se pudo evidenciar como los resultados de los estudias mejoraron, debido a que llegaron a la solución en un menor tiempo y tuvieron mayor comprensión, en comparación con los estudiantes que no lo implementaron (Valdez, 2013).

Ahora bien, también se evidencio por medio de la programación, que es posible resolver sistemas de ecuaciones lineales, tal es el caso de Excel, el cual después de haber sido programado, solo es cuestión de introducir valores y se obtendrá el resultado. (González, y Llamas, 2015).

Ahora, siguiendo con la línea de la transversalidad, se evidencio que, por medio de las historias narrativas, se puede contribuir a construir el conocimiento de los estudiantes, de esta forma ellos logran tener una comprensión mayor de las temáticas debido a que lo relacionan con cosas concretas, sin embargo, es preciso que el docente adapte los textos según las necesidades de aprendizajes (Frabetti, 2009).

Ahora bien, otra herramienta frecuente en la educación matemática, es la resolución de problemas contextualizados, los cuales didácticamentepermiten comprender mejor la importancia de los sistemas de ecuaciones lineales al mismo tiempo que dan 
cabida para diferentes formas de representación de los mismos (Camarena y Trejo, 2009). Así mediante representaciones de su medio hacen uso de sus conocimientos previos para lograr llegar a respuestas más coherentes, pero también muy significativas (Trejo y Camarena, 2010). Sin embargo, es necesario que se oriente al estudiante acerca de la resolución de estos, proponiéndole estrategias y herramientas que le puedan orientar en este proceso, así pues, hay que ir más allá del planteamiento, sino que mejor aún hay que acompañarlos en la iniciación de esta valiosa estrategia de aprendizaje (Urdiain, 2006).

Otro elemento no menos relevante en el proceso educativo es la motivación del estudiante y esta se puede estimular por medio de juegos, debido a que al mismo tiempo que se divierten van relacionando variables y elementos que se emplean en su diario vivir con los conceptos matemáticos trabajados (Gutiérrez, 2017). Así pues, se puede desarrollar el conocimiento en los estudiantes, por medio de problemas los cuales el estudiante solucionara por medio de sus habilidades y fortaleciendo sus aprendizajes, al mismo tiempo que se divierte (Villagrán y Olfos, 2001).

Finalmente es necesario destacar que la evaluación no ha de quedarse como un elemento sumativo, sino por el contrario, ha de ser un proceso formativo que le permita al estudiante tener el control de su proceso y lo lleve a reflexionar sobre lo que debe mejorar. (de Galindo y de García, 2010).

\section{Conclusiones}

La solución de los sistemas de ecuaciones lineales es una temática fundamental, no solo en el área de las matemáticas, sino también en otros campos. Así pues, es de vital importancia lograr una completa interpretación del tema por parte de los estudiantes. Sin embargo, esto ha sido algo complejo debido a que generalmente el proceso formativo entorno a esta temática se realiza de forma procedimental y casi que por imitación.
Ahora bien, aparecen diferentes alternativas didácticas que ofrecen nuevas formas de enseñanza y proponen recursos que anteriormente no se tenían para facilitar el aprendizaje, así pues se recomiendan opciones como enseñar la temática con un proceso transversal por medio de juegos, recursos digitales, lecturas e incluso ecuaciones químicas, de tal forma que el estudiante logre asociar el conocimiento, con elementos del entorno, descubriendo así, que realizado tiene repercusión en su contexto y desarrollando así un aprendizaje significativo.

Por otro lado, se evidencio que gran mayoría de los errores y dificultades que tienen los estudiantes, se deben a la falta de bases y conceptualización matemática, lo cual se estima que es causado por la falta de profundización en los fundamentos algebraicos, al igual que en el desarrollo de los procesos mentales, tanto así, que se evidenciaron falencias incluso en estudiantes universitarios. Por lo cual se hace necesario una formación en los fundamentos algebraicos desde la educación primaria, aunque para ello es necesario una pertinente capacitación docente.

\section{Referencias}

Abrate, R., Font, V., \& Pochulu, M. (2008).

Obstáculos y dificultades que ocasionan resolución de ecuaciones. Proyecciones, 6(2), 49-56.

Alfonso, B. G., García, M. T. S., \& Gabarda, I. H. (2016).Problemas Descriptivos de fracciones Boletim de Educação Matemática, 30(55), 586604.

Alurralde, F., \& Ibarra, L. (2008).El uso de las letras en álgebra: Análisis de una evaluación de estudiantes de primer año de ingeniería. Revista de educación matemática.

Arroyo, G. C. (2014).Dificultades en el aprendizaje de problemas que se modelan con ecuaciones lineales: El caso de estudiantes de octavo nivel de un colegio de Heredia. Uniciencia, 28(2), 1544.

Benítez, N. S., \& Drouhard, J. P. (2015). Una mirada epistemográfica sobre el rol de las dificultades 
algebraicas ligadas al estudio de funciones en el ingreso a la universidad. In IV Jornadas de Enseñanza e Investigación Educativa en el campo de las Ciencias Exactas y Naturales.

Camarena, P., \& Trejo, E. (2009).Problemas contextualizados: una estrategia didáctica para aprender matemáticas.

Cambriglia, V. (2008).Procesos de generalización en matemática en la transición primaria-media. Revista de educación matemática.

Castellón, R. E. R., Sánchez, E. L., Moreno, M. G.A., \& Páez, R. A. B. (2003).Estudio del dominio del lenguaje algebraico que prevalece entre alumnos de nuevo ingreso, universidad autónoma de baja California- Instituto Tecnológico de Mexicali.

Castro, W., Godino, J. D., \& Rivas, M. (2011). Razonamiento algebraico en educación primaria: Un reto para la formación inicial de profesores. UNION Revista Iberoamericana de Educación Matemática, 25, 73-88.

Cedro, W. L., \& Moura, M. (2010).Experimento didáctico: un camino metodológico para la investigación en la educación matemática. Unión: Revista Iberoamericana de Educación Matemática, 22, 53-63.

Córdoba, L., Díaz, M. E., Haye, E. E., \& Montenegro, F. (2013).Dificultades de los alumnos para articular representaciones gráficas y algebraicas de funciones lineales y cuadráticas.

Costa, W. R. (2010).Investigando a conversão da escrita natural para registros em escrita algébrica em problemas envolvendo equações de primeiro grau (Master's thesis, Universidade Federal de Pernambuco).

Dalcin, M., \& Olave, M. (2007).Ecuaciones de primer grado: su historia.

De Galindo, S. G., \& de García, P. V. (2010). Metacognición: Diseño de un material curricular para aulas multitudinarias. Revista electrónica de investigación en educación en ciencias, 5(2), 58-69.

De Herrero, S. M. S. (2004). Sistemas de ecuaciones lineales: una secuencia didáctica. Revista
Latinoamericana de Investigación en Matemática Educativa, RELIME, 7(1), 49-78.

Del Puerto, S., Minnaard, C. L., \& Seminara, S. (2006).Análisis de los errores: una valiosa fuente de información acerca del aprendizaje de las Matemáticas. Revista Iberoamericana de educación, 38.

Díaz Ruiz, B. (2016).Análisis de errores en las pruebas de acceso a la Universidad: el caso de los sistemas de ecuaciones lineales.

Duarte, P. C. X. (2013).A linguagem no ensino de matemática. Nucleus, 10(1).

Esquinas, A. M. (2009).Dificultades de aprendizaje del lenguaje algebraico: del símbolo a la formalización algebraica: aplicación a la práctica docente (Doctoral dissertation, Universidad Complutense de Madrid, Servicio de Publicaciones).

Figueroa Vera, R. E. (2013).Resolución de problemas con sistemas de ecuaciones lineales con dos variables: una propuesta para el cuarto año de secundaria desde la teoría de situaciones didácticas.

Frabetti, C. (2009).Literatura y matemáticas. Uno: Revista de didáctica de las matematicas, (50), 42-46.

Gaisman, M. T. (2009).El uso de la modelación en la enseñanza de las matemáticas. Innovación educativa, 9(46), 75-87.

García-García j. (2019).Strategies in solving algebraic problems in an intercultural context in Higher Education. Bolema: Boletim de Educação Matemática, 33(63), 205-225.

González, D., \& Roa, S. (2015).Los sistemas de ecuaciones lineales: evidencias del tránsito entre los modos de pensamiento en estudiantes universitarios.

González, F. J. P., \& Llamas, M. D. C. G. (2015). Resolución de sistemas de ecuaciones lineales con Excel. Anales de ASEPUMA, (23), 3.

Gutiérrez, A., \& De, T. D. J. (2017).Utilización de los juegos como herramienta didáctica para 
potenciar el proceso de la solución de ecuaciones de primer grado con una incógnita (Master's thesis, Universidad del Norte).

Iribar, N. G., \& Rouseau, M. L. (2009).El tratamiento de errores en el aprendizaje de procedimientos algebraicos. EduSol, 9(26), 73-85.

Issakova, M. (2006).Comparison of student errors made during linear equation solving on paper and in interactive learning environment. In Dresden International Symposium on Technology and its Integration into Mathematics Education.

Jara, M. A. R., Lorca, A. M., Lorca, J. M., Saldias, P. V., \& Leo, M. E. D. V. (2019). Construcción cognitiva del conjunto solución de un sistema de ecuaciones lineales con dos incógnitas. Enseñanza de las ciencias: revista de investigación y experiencias didácticas, 37(1), 71-92.

Kiener, F. (2015).Una propuesta para iniciar el trabajo algebraico en la escuela primaria: el caso de los gogos. Epsilon: Revista de la Sociedad Andaluza de Educación Matemática" Thales", (90), 39-48.

Linares, P., Ramos, A., Sánchez, P., Sarabia, A., \& Vitoriano, B. (2001).Modelos matemáticos de optimización. Madrid: Universidad Pontificia de Comillas.

Martínez, L., Rincón, E. G., \& Domínguez, Á. (2011). El juego y el aprendizaje cooperativo en la enseñanza de las ecuaciones de primer grado.

Medrano, A., \& Flores-Macías, R. D. C. (2018). Álgebra Temprana como herramienta de análisis y comprensión de problemas aritméticos en primaria.

Model, S. L. (2005). Dificuldades de alunos com a simbologia matemática.

Oaxaca, J., De la Cruz, J, Sánchez, J. (2009). Dificultades en el tránsito del razonamiento sintético-geométrico al analítico-aritmético en la solución de sistemas de ecuaciones lineales. Trabajo presentado en el Primer Congreso internacional sobre la Enseñanza de las Matemáticas, Cuautitlán Izcali, México.

Ochoviet, C. (2009).Sobre el concepto de solución de un sistema de ecuaciones lineales con dos incógnitas Tesis (Doctorado en Matemática Educativa). CICATA-IPN, Ciudad de México, México.

Otero, M. R., Papini, C., \& Elichiribehety, I. (2016). Las representaciones mentales y la resolución de un problema: Un estudio exploratorio. Investigaçoes em Ensino de Ciencias, 3(1), 4760.

Pérez, M., Diego-Mantecón, J. M., Polo, I., \& González, M. J. (2019).Causas de los errores en la resolución de ecuaciones lineales con una incógnita. PNA, 13(2), 84-103.

Ramírez, G., Chavarría, J., Barahona, C., \& Mora, M. (2009).Análisis de las conceptualizaciones erróneas en conceptos de geometría y sistemas de ecuaciones: un estudio con estudiantes universitarios de primer ingreso. Revista Digital: Matemática, Educación e Internet, 10(1).

Rey, G., \& Sastre, P. (2013).Lenguaje Matemático: análisis diagnóstico en estudiantes que ingresan a la universidad.

Rodríguez, E., \& Fernández, C. M. V. (2010). Significado institucional referencial de la función afín y ecuación lineal en la economía. Gestión y Gerencia, 4(2), 63-87.

Romero, L. R. (2004).Evaluación de competencias matemáticas: proyecto PISA/OCDE 2003. In Investigación en educación matemática: Octavo Simposio de la Sociedad Española de Investigación en Educación Matemática (SEIEM): A Coruña, 9-11 septiembre 2004 (pp. 89-102). Servizo de Publicacións.

Sánchez, N. A. (2014).Análisis de errores asociados a la resolución de ecuaciones de primer grado. una aproximación desde la zona de desarrollo próximo.

Sanjosé, V., Valenzuela, T., Fortes, M. C., \& SolazPortolés, J. (2007).Dificultades algebraicas en la resolución de problemas por transferencia. Revista electrónica de Enseñanza de las Ciencias, 6(3), 538-561.

Suárez, J. G., Alex, I. S., \& Gómez, J. L. L. (2014). El Uso de Las Letras como Fuente de Errores 
de Estudiantes Universitarios en la Resolución de Tareas Algebraicas. Bolema: Boletim de Educação Matemática, 28(50), 1545-1566.

Trejo, E., \& Camarena, P. (2010).Análisis cognitivo de los alumnos al resolver problemas contextualizados.

Trejo, E., \& Camarena, P. (2011).Análisis cognitivo de situaciones problema con sistemas de ecuaciones algebraicas en el contexto del balance de materia. Educación matemática, 23(2), 65-90.

Urdiain, I. E. (2006).Matemáticas resolución de problemas. Navarra: Fondo de publicaciones del gobierno de Navarra.

Valdez, S. K. L. (2013).Uso de software especializado para incrementar el aprendizaje de solución de ecuaciones lineales de una variable.

Valencia, V. (2012) Revisión documental en el proceso de investigación." Universidad Tecnológica de Pereira. Bogotá, Colombia.

Villagrán, E., \& Olfos, R. (2001)Actividades lúdicas y juegos en la iniciación al álgebra. Revista Integra, 5, 39-50. 